\title{
VAC.25 - Ebola vaccine induced arthritis: Gene predictors from a Random Forest Model.
}

Patrícia Gonzalez-Dias ${ }^{1 *}$; Helder I Nakaya ${ }^{1}$.

1USP - Universidade de São Paulo.

Introduction: Hemorrhagic fever induced by the Ebola virus has caused over 11.300 deaths in the 2014 outbreak. Extraordinary efforts from the World Health Organization and other institutions were made to contain the spread of the disease by supporting development of vaccines. VSV-ZEBOV is a recombinant vesicular stomatitis virus (VSV) vaccine that has been shown to be immunogenic, safe and protective. However, VSV-ZEBOV also causes some adverse effects, including subjective and objective fever, chills, myalgia and arthritis, which is the most severe.

Objective: Identification of reactogenicity and/or protection predictors in vaccinated subjects to avoid arthritis' adverse effect in further vaccinated populations.

Methodology: We implemented a Random Forest model based on gene expression data from 63 vaccinated subjects of the Geneva Cohort to predict the risk of arthritis induced by VSVZEBOV. Gene expression data was assessed by dual-color Reverse Transcriptase Multiplex Ligation-dependent Probe Amplification (dcRT-MLPA) assay. Baseline gene expression values (Day 0) were used as predictors of adverse events.

Random forest parameters were set as following: 1.000 trees and 10.000 permutations, with additional parameters set to their default values. The mean decrease of Gini index (MDGI) was obtained for each feature (gene).

Results: Our model identified LAG3 (MDGI = 0.663), TAP1 (0.625), GBP1 (0.528), NLRP3 (0.513), DSE (0.375) as the top important predictive arthritis genes. These genes have studies relating them to arthritis. For example, Lymphocyte-activation gene 3 was the most predictive of arthritis induced by VSV-ZEBOV. Recent reports have shown a role for LAG-3 in arthritis severity reduction. The authors showed that the use of LAG3 Treg-of-B-cells significantly reduced the clinical severity and inflammatory response in Collagen-Induced Arthritis (CIA) mice, supporting a novel therapeutic role of LAG3 p Treg-of-B cells in Rheumatoid Arthritis (RA) and other autoimmune diseases. In addiction, another research group has shown that IL-10-producing LAG3 + Tregs are associated with the immunopathology and therapeutic response in RA.

Conclusion: Thus, our results shows the effectiveness of Random Forest as a predictive model to select features related to vaccination induced arthritis, and may in the future aid the selection of patients who can receive the VSV-ZEBOV with reduced adverse events.

Keywords: Adverse Events; Machine Learning; Ebola Virus Disease 